\title{
Edge Sensing-Enabled Multistage Hierarchical Clustering Deredundancy Algorithm in WSNs
}

\author{
Rongbo Zhu $\mathbb{D}^{1},{ }^{1}$ Mai Yu, ${ }^{1}$ Yuanli Li, ${ }^{1}$ Jun Wang, ${ }^{1}$ and Lu Liu ${ }^{2}$ \\ ${ }^{1}$ College of Computer Science, South-Central University for Nationalities, Wuhan 430074, China \\ ${ }^{2}$ School of Informatics, University of Leicester, Leicester, UK \\ Correspondence should be addressed to Rongbo Zhu; rbzhu@mail.scuec.edu.cn
}

Received 25 November 2020; Revised 31 January 2021; Accepted 22 February 2021; Published 12 March 2021

Academic Editor: Shaohua Wan

Copyright (C) 2021 Rongbo Zhu et al. This is an open access article distributed under the Creative Commons Attribution License, which permits unrestricted use, distribution, and reproduction in any medium, provided the original work is properly cited.

\begin{abstract}
Due to the defects caused by limited energy, storage capacity, and computing ability, the increasing amount of sensing data has become a challenge in wireless sensor networks (WSNs). To decrease the additional power consumption and extend the lifetime of a WSN, a multistage hierarchical clustering deredundancy algorithm is proposed. In the first stage, a dual-metric distance is employed, and redundant nodes are preliminarily identified by the improved $k$-means algorithm to obtain clusters of similar nodes. Then, a Gaussian hybrid clustering classification algorithm is presented to implement data similarity clustering for edge sensing data in the second stage. In the third stage, the clustered sensing data is randomly weighted to deduplicate the spatial correlation data. Detailed experimental results show that, compared with the existing schemes, the proposed deredundancy algorithm can achieve better performance in terms of redundant data ratio, energy consumption, and network lifetime.
\end{abstract}

\section{Introduction}

Wireless sensor networks (WSNs) are common in people's lives and are widely used in various fields $[1,2]$. WSNs are deployed in different areas to monitor environments and objects, such as temperature, humidity, and seismic events $[3,4]$. To obtain accurate sensing data for events, a large number of sensors are utilized to collect the edge sensing data and transmit the data to an aggregation/sink node in a highfrequency manner. In general, edge sensing data have a high spatial-temporal correlation and contain considerable redundant information [5, 6]. Additionally, the transmission of redundant data leads to unnecessary energy consumption and bandwidth costs, which increase the overhead and decrease WSN lifetimes. Therefore, reducing redundant data and the transmission energy consumption to extend network lifetimes becomes a key issue in WSNs.

To reduce redundant data effectively, the existing work concentrates on two aspects: optimizing sensing data and predicting sensing data. On the one hand, the former is aimed at reducing redundant sensing data with some optimized schemes. Considering the constrained resources in WSNs, a spatial-temporal correlation data reduction scheme was proposed to determine the optimal sampling strategy for the deployed sensor nodes (SNs) [7]; the strategy reduces the overall sampling/transmission rates while preserving the quality of the data. Considering that the data volume increases with unexpected ratios in WSNs, an integrated divide and conquer method with an enhanced $k$-means scheme was proposed [8], which removes redundant data from the collected measures. To save the limited energy of WSNs, a data transmission (Dat) protocol, which can reduce the data transmission cost inside each sensor node by removing redundant data to save energy while maintaining a suitable level of accuracy in the received readings at the sink, was presented [9]. To conserve energy and enhance the lifetime of a WSN, reducing the amount of data communicated by exploiting the temporal and spatial correlations of the sensed data is a suitable approach. An energy-efficient semantic clustering model was proposed to mitigate the high-energy consumption problem in a clustered WSN [10]. To reduce the energy consumed during data transmission, an adaptive data reduction method, which is based on a convex combination of two decoupled least-mean-square windowed filters, was proposed [11]. 
On the other hand, the prediction-based scheme tries to reduce sensing data with forecasting schemes. To improve data processing efficiency, a distributed data prediction model based on least squares, which tries to use a data predictionbased filtering scheme, was proposed to decrease transmission data [12]. Alduais et al. [13] presented an updating frequency metric, which is defined as the frequency of updating the model reference parameters during data collection, to evaluate the performance of different multivariate data reduction models for WSNs. To schedule data communications between SNs and a sink to reduce power usage with the aim of maximizing the network lifetime, a prediction-based data communication scheme, which utilizes the hierarchical least-mean-square adaptive filter to predict the measured values both at the source and at the sink, was presented [14].

Although the schemes mentioned above provide efficient solutions to reduce redundant sensing data in WSNs, the following defects still need to be addressed comprehensively. Firstly, a large range of edge sensing data and errors in local best values can lead to local characteristics being lost. Then, the errors of sensing data will result in a similarity threshold failure problem. And the existing distance-based correlation reducing redundant sensing data schemes, which only consider the spatial corrections of sensing event and omit the temporal correlations of sensing data, tend to degrade the accuracy of sensing data. Furthermore, prediction-based schemes require relatively long-term data sensing and processing abilities, which increase the burden of resourcelimited sensors and decrease the lifetime of WSNs. Hence, it is necessary to consider both spatial and temporal correlations and location and data similarity clustering to decrease the sensing data transmission and processing. Focusing on the issue mentioned above, this paper explores the sensing data deredundancy problem to decrease energy consumption and extend the lifetime of a WSN.

The main contributions of this paper are summarized as follows:

(1) A multistage hierarchical clustering similarity deredundancy (MHCSD) algorithm is proposed to reduce the power consumption and extend the lifetime of a WSN. MHCSD considers both spatial and temporal correlations and location and data similarity clustering to overcome the accuracy degradation of sensing data

(2) A dual-metric distance is employed in the first stage, and an improved $k$-means algorithm is proposed to judge the similarity of nodes based on the dualmetric distance in sinks. A Gaussian hybrid clustering algorithm is presented to judge the similarity of edge sensing data within the same cluster and can improve the similarity accuracy and the deredundancy ratio. The clustered sensing data are randomly weighted to further deduplicate the spatial correlation data in the third stage

The remainder of this paper is organized as follows. Related work is explored in Section 2. Section 3 presents the proposed multistage hierarchical clustering deredun- dancy algorithm in WSNs. Section 4 shows the experimental results, which verify the proposed scheme. The paper is concluded in Section 5 finally.

\section{Related Work}

Although a large amount of application-specific data are generated in WSNs, most of the sensing data detected by sensors are redundant. Processing and transmitting massive superfluous data can lead to additional power consumption and greatly decrease network lifetime $[15,16]$. To improve data processing performance, a path merging protocol, which supports partial discrete wavelet transform-based compression schemes to reduce redundant data transmission in a significant manner through the appropriate aggregation of data packets from merging paths, was proposed in [17]. To manage energy-efficient data collections in WSNs, a dataaware energy conservation scheme and prediction-based data collection framework were proposed to reduce data transmission [18], where the inherent correlation between the consecutive observations of SNs and the data similarity measures between the neighboring SNs are utilized.

Considering that the data volume in WSNs is quickly increasing, a hybrid-stream big data analytics model, which utilizes a multidimensional convolutional neural network $(\mathrm{CNN})$, minimal correlation model, and minimal redundancy model to optimize data processing, is proposed to perform big data analysis [19]. To provide a complete description of an environment and make a robust decision, a redundancy removal strategy, which mines the spatial and temporal data from collected data to select the appropriate information before forwarding to a base station or a cluster head $(\mathrm{CH})$ in a WSN, is proposed [20]. To avoid generating, transmitting, and storing unwanted data from redundant messages, an immunization-based redundancy elimination scheme, which independently selects the correct number of acknowledgment frames distributed to respond to variations in the amount of redundant data in a dynamic fashion, was proposed [21]. An image fusion method was proposed based on histogram similarity and multiview weighted sparse representations [22].

By introducing histogram similarity, different weights are given to low-resolution high-frequency components and source image high-frequency components, and complementary information is effectively used. Diwakaran et al. [18] used the inherent correlations between the continuous observations of SNs and the data similarity measures of adjacent $\mathrm{SNs}$ to reduce data transmission. A new model based on monkey tree search behavior inspired by fauna was explored in [23], and the fuzzy reasoning mechanism was used to complete data collection and dissemination. Rida et al. [24] utilized data aggregation techniques based on the Euclidean distance to reduce similar data. Lin et al. [25] proposed a semantic data annotation method based on semantics. A data clustering method, which groups homogeneous data into clusters and then performs data reduction by selecting the average value of each cluster, was proposed based on histograms for data reduction [26]. 
Additionally, to address the problem of redundant data collected by sensors, data aggregation and semaphore processing based on similar functions are applied in WSNs, and SNs are aggregated with a palm tree method [27]. Wan et al. [28] proposed a similar sensory data aggregation scheme based on fuzzy $c$-means. A spatial-temporal correlation search mechanism between SNs based on the Euclidean distance is proposed [29]. An energy-saving redundant traffic handling scheme, which utilizes short beacon information to process redundant packets generated in area-based routing, was presented in [30]. The parameter estimation problem was considered in [31], and two censoring algorithms were proposed to enable SNs to transmit sampled data based on local decision-making. The dual prediction scheme is used to reduce the transmission between cluster nodes and $\mathrm{CHs}$, while the data compression scheme is used to reduce the traffic between $\mathrm{CHs}$ and sink nodes [32]. A low redundancy data acquisition scheme, which selects some nodes for data detection and transmits less data to $\mathrm{CHs}$, was proposed based on matrix completion [33]. To reduce transmitted data, a differential data processing (DDP) method was proposed in [34].

Although there are many effective deredundancy processing schemes in WSNs, the following limitations still need to be addressed. Data correlation analysis does not consider homologous data, which can result in a lower deredundancy ratio and loss of local characteristics. Furthermore, unconscionable deredundancy can degrade the accuracy of sensing data. Focusing on filling this gap, this paper proposes a multiphase hierarchical clustering similarity deredundancy algorithm to overcome the limitations mentioned above.

\section{Proposed Scheme}

3.1. System Model. There is a set $S=\left\{s_{1}, s_{2}, \cdots, s_{n}\right\}$ composed of $n$ SNs. And the edge sensor nodes will collect data. The system model is shown in Figure 1, and Table 1 lists the notation that we use in the paper.

The sink calculates the similar distances between nodes according to the coordinates of the nodes and divides the nodes into $K$ clusters according to the similar distances $S=$ $\left\{C_{1}, C_{2}, C_{3}, \cdots, C_{K}\right\}$, where $C_{i} \cap C_{j}=\varnothing(i \neq j) . \mathrm{CH}_{i}$ of each cluster collects the sensing data generated by the nodes in the cluster at time $t_{j}$ as set $D_{\mathrm{CH}_{h}}\left(t_{j}\right)=\left\{x_{1}\left(t_{j}\right), x_{2}\left(t_{j}\right), \cdots, x_{n}\left(t_{j}\right)\right\}$. Gaussian mixed clustering is adopted to classify the collected data into similar clusters and then classifies the nodes in the cluster as $C_{i}=\left\{C_{i, 1}, C_{i, 2}, C_{i, 3}, \cdots, C_{i, m}\right\}, C_{i, j}=\left\{s_{i, j, 1}\right.$, $\left.s_{i, j, 2}, \cdots, s_{i, j, k}\right\}$, where $1 \leq i \leq K, 1 \leq j \leq m$.

3.2. Deredundancy Algorithm. The proposed MHCSD algorithm includes three stages: the local clustering stage, the similar data clustering stage, and the data deredundancy processing stage. In addition, the framework of MHCSD is shown in Figure 2. In the first stage, the sink will perform the improved $k$-means clustering algorithm, which clusters similar nodes according to the spatial position coordinates of the nodes. Then, in the second stage, $\mathrm{CHs}$ adopt the Gaussian hybrid clustering algorithm to further seek similar clusters. In the third stage, based on the maximum time threshold, the SNs utilize an adaptive step length in the data deredundancy scheme (TCDA) to eliminate duplicate sensing data with spatial-temporal correlations.

3.2.1. Similarity of $S N s$. In the first stage, the node similarity analysis is performed according to the node position coordinates in the sink. To delete duplicate edge sensing data effectively, local clustering needs a precise similarity measure among nodes and sensing data. Among various distance metrics, the Euclidean distance may be the most commonly used in data processing. However, the Euclidean distance only describes the amplitude difference between two eigenvectors, and the Euclidean distance of two feature vectors with different shapes may be smaller than that of feature vectors with similar shapes. To overcome the defect in the Euclidean distance, a dual-metric similarity distance $D(i, j)$ is employed:

$$
D(i, j)=D_{E}(i, j)+\beta D_{P}(i, j)
$$

where $D_{E}(i, j)$ is the Euclidean distance, $D_{P}(i, j)$ is the Pearson correlation distance, and $\beta$ is a scale factor that indicates the influence of $D_{P}(i, j)$ on the weight of $D(i, j)$. In addition, we have

$$
\begin{aligned}
& D_{E}(i, j)=\sqrt{\sum_{h=1}^{h}\left(l_{i, h}-l_{j, h}\right)^{2}}, \\
& D_{P}(i, j)=\frac{1}{2}\left(1-\frac{\sum_{h=1}^{h}\left(l_{i, h}-\bar{l}_{i, h}\right)\left(l_{j, h}-\bar{l}_{j, h}\right)}{\sqrt{\sum_{h=1}^{h}\left(l_{i, h}-\bar{l}_{i, h}\right)^{2} \sum_{h=1}^{h}\left(l_{j, h}-\bar{l}_{j, h}\right)^{2}}}\right) .
\end{aligned}
$$

The dual-metric similarity distance $D(i, j)$ meets three distance characteristics: positivity, symmetry, and reflexivity. In terms of $D(i, j)$, any active feature vector pair can be compared from both the amplitude of the Euclidean distance and the change in the shape of the related distance.

For $S=\left\{s_{1}, s_{2}, \cdots, s_{n}\right\}$, the spatial position coordinate $l_{i}$ of node $s_{i}$ is $\left(x_{i}, y_{i}\right)$. The sink performs the improved $k$-means algorithm as shown in Algorithm 1.

According to coordinate position set $L=\left\{l_{1}, l_{2}, \cdots, l_{n}\right\}, n$ nodes are classified in $K$ disjoint subsets $C_{i} . C=\left\{C_{1}, C_{2}\right.$, $\left.\cdots, C_{K}\right\}$ and $C_{1} \cup C_{2} \cup \cdots \cup C_{K}=S$, where $C_{i} \neq \varnothing$ and $C_{i} \cap$ $C_{j}=\varnothing, i \neq j$. In addition, the minimum squared error $e$ is defined as

$$
e=\sum_{i=1}^{K} \sum_{l \in C_{i}}\left\|l-\bar{\mu}_{i}\right\|_{2}^{2}
$$

where $\bar{\mu}_{i}=\left(1 /\left|C_{i}\right|\right) \sum_{l \in C_{i}} l$ is the mean vector of cluster $C_{i}$

3.2.2. Similarity of Sensing Data. After clustering the similar nodes by the spatial positions in the first stage, to refine redundant judgments of nodes, the Gaussian hybrid 


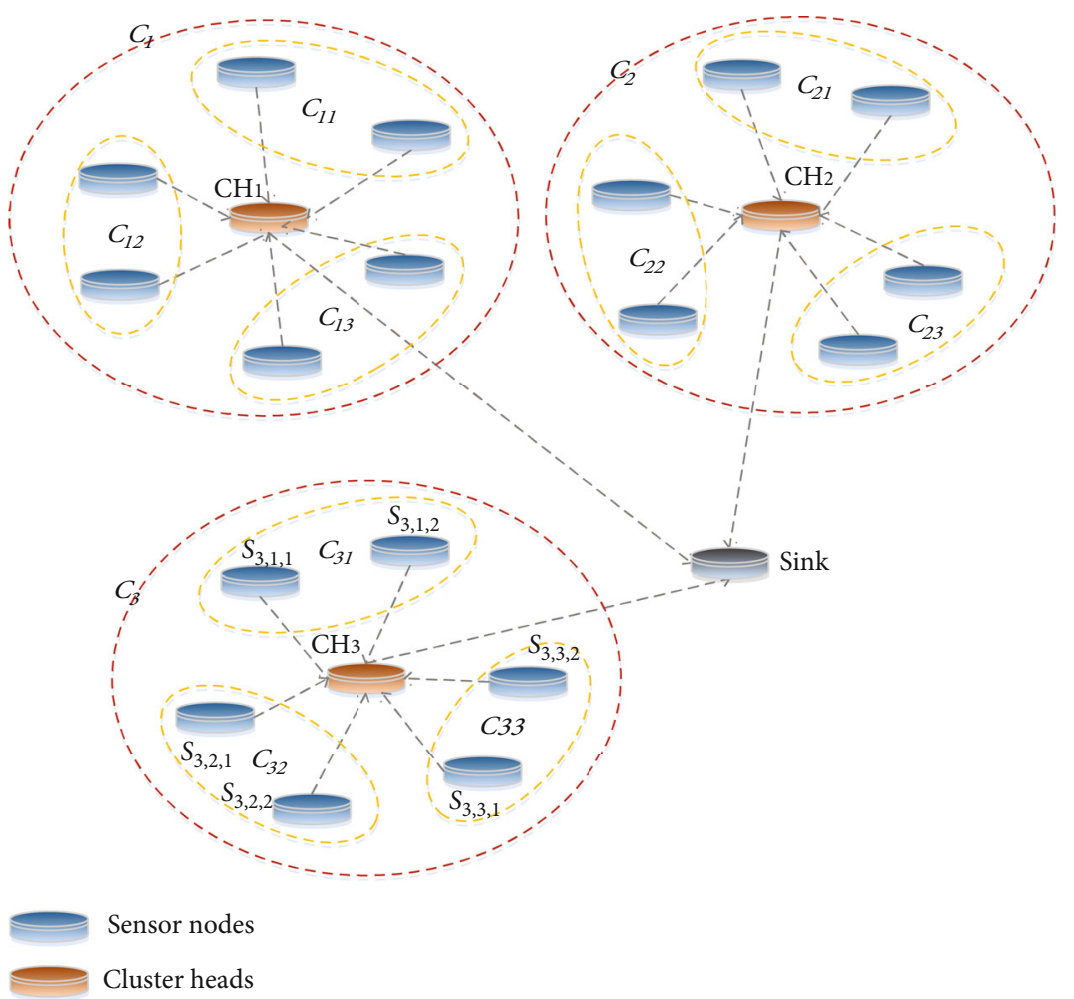

Figure 1: System model.

clustering algorithm is adopted in the second stage and is shown in Algorithm 2.

To further analyze the similarity of the data collected simultaneously within cluster $C_{i}$, the probabilistic model is used to analyze and describe the prototype data in Gaussian hybrid clustering. The cluster division is mainly determined by the posterior probability corresponding to the prototype. The Gaussian distribution is defined as the random variable $x$ in the $n$-dimensional sample space $X$, and its probability density function $p(x)$ is defined as

$$
p(x)=\frac{1}{(2 \pi)^{n / 2}|\aleph|^{1 / 2}} e^{-1 / 2(x-\mu)^{T} \aleph^{-1}(x-\mu)},
$$

where $\mu$ represents the $n$-dimensional mean vector and $\aleph$ denotes the $n \times n$ covariance matrix. Since the Gaussian distribution is determined by the mean vector $\mu$ and the covariance matrix $\aleph$, for the convenience of description, the probability density function for the dependence of the Gaussian distribution on the corresponding parameters is expressed as $p(x \mid \mu, \aleph)$. The Gaussian mixture distribution $p_{M}$ is

$$
p_{M}=\sum_{i=1}^{K_{1}} \alpha_{i} \bullet p\left(x \mid \mu_{i}, \aleph_{i}\right)
$$

where $\mu_{i}$ and $\aleph_{i}$ are the parameters of the $i$ th Gaussian mixed component, $\alpha_{i}>0$ is the corresponding mixing coefficient, and $\sum_{i=1}^{K_{1}} \alpha_{i}=1$. $p_{M}$ consists of $K_{1}$ mixed com- ponents, and each mixed component corresponds to a Gaussian distribution.

For $S$ composed of $K$ clusters, data generated by a cluster can be expressed as a set $X=\left\{X_{1}, X_{2}, \cdots, X_{n}\right\}$, and $X_{i}=\left\{x_{i}\left(t_{1}\right), x_{i}\left(t_{2}\right), \cdots, x_{i}\left(t_{2}\right)\right\}$, where $1 \leq i \leq n$ is the set of time series generated by the sensor node $s_{i}$ every $T$ seconds. In the WSN, each $\mathrm{CH}$ continues to classify the correlated data of the nodes in the cluster, and through the Gaussian hybrid clustering algorithm, the data collection $D_{\mathrm{CH}_{h}}\left(t_{j}\right)$ $=\left\{x_{1}\left(t_{j}\right), x_{2}\left(t_{j}\right), \cdots, x_{z}\left(t_{j}\right)\right\}$ in similar clusters of the same spatial nodes is simultaneously divided into $K_{1}$ clusters, where $1 \leq j \& \& 1 \leq h \leq K_{1}$.

It is assumed that the random variable $z_{j_{1}} \in\left\{1,2, \cdots, K_{1}\right\}$ represents the Gaussian mixture component of the sensing data $x_{j_{1}}\left(t_{j}\right)$ of node $j_{1}$. The prior probability $P\left(z_{j_{1}}=i\right)$ of $z_{j_{1}}$ corresponds to $\alpha_{i}\left(i=1,2, \cdots, K_{1}\right)$. According to Bayes' theorem, the posterior distribution of $z_{j_{1}}$ corresponds to

$$
\begin{aligned}
p_{M}\left(z_{j_{1}}=i \mid x_{j_{1}}\left(t_{j}\right)\right) & =\frac{P\left(z_{j_{1}}=i\right) \bullet p_{M}\left(x_{j_{1}}\left(t_{j}\right) \mid z_{j_{1}}=i\right)}{p_{M}\left(x_{j_{1}}\left(t_{j}\right)\right)} \\
& =\frac{\alpha_{i} \bullet p\left(x_{j_{1}}\left(t_{j}\right) \mid \mu_{i}, \aleph_{i}\right)}{\sum_{l=1}^{k} \alpha_{l} \bullet p\left(x_{j_{1}}\left(t_{j}\right) \mid \mu_{l}, \aleph_{l}\right)} .
\end{aligned}
$$

$p_{M}\left(z_{j_{1}}=i \mid x_{j_{1}}\left(t_{j}\right)\right)$ is expressed as sample $x_{j_{1}}\left(t_{j}\right)$ generated by the $i$ th Gaussian mixture composition of the a posteriori probability, expressed as $\gamma_{j_{1} i}\left(i=1,2, \cdots, K_{1}\right)$. 
TABle 1: Notation.

\begin{tabular}{|c|c|}
\hline Symbol & Description \\
\hline$K$ & Number of similar clusters \\
\hline$\beta$ & $\begin{array}{l}\text { The effect of } D_{P}(i, j) \text { on the weight of } D(i, j) \text {, weight } \\
\text { scaling factor }\end{array}$ \\
\hline$n$ & Node size \\
\hline$m$ & Number of similar clusters \\
\hline$K_{1}$ & Number of data similar clusters \\
\hline$\alpha_{i}$ & Gaussian mixture coefficient \\
\hline$z_{j_{1}}$ & Gaussian mixture components \\
\hline$\gamma_{j_{1}, i}$ & The posterior probability of the Gaussian mixture \\
\hline$\rho_{j_{1}}$ & Cluster markers of sample $x_{j_{1}}\left(t_{j}\right)$ \\
\hline$\mu_{i}$ & The average of the components \\
\hline$\rho$ & Lagrange multiplier \\
\hline$\beta_{i}$ & Random weighting factor \\
\hline$e$ & Minimizing the squared error in clustering of $k$-means \\
\hline$E_{\text {elec }}$ & $\begin{array}{l}\text { Power consumption of a circuit for sending or receiving } \\
\text { data }\end{array}$ \\
\hline$\varepsilon_{m p}, \varepsilon_{f s}$ & Energy consumption of signal amplifiers \\
\hline$E_{P}$ & Energy consumption per unit of data \\
\hline$D_{E}(i, j)$ & Euclidean distance \\
\hline$D_{P}(i, j)$ & Pearson correlation distance \\
\hline$D(i, j)$ & Spatial similarity distance \\
\hline $\bar{\mu}_{i}$ & The mean of cluster $C_{i}$ \\
\hline$\aleph$ & $n \times n$ covariance matrix \\
\hline$\rho_{j_{1}}$ & Cluster markers \\
\hline$D_{C_{i, j_{1}}}\left(t_{j}\right)$ & $\begin{array}{l}\text { The random weighted deredundancy of sensing data in } \\
\qquad C_{i, j_{1}}\end{array}$ \\
\hline
\end{tabular}

After the Gaussian mixture distribution, the cluster becomes the sample set $D_{\mathrm{CH}_{h}}\left(t_{j}\right)$ divided into $K_{1}$ subclusters and expressed as set $C=\left\{C_{i, 1}, C_{i, 2}, C_{i, 3}, \cdots, C_{i, K_{1}}\right\}(0<i$ $\left.\leq K_{1}\right)$; the cluster markers $\rho_{j_{1}}$ of each sample $x_{j_{1}}\left(t_{j}\right)$ are defined as follows:

$$
\rho_{j_{1}}=\underset{i \in(1,2, \cdots, 3)}{\operatorname{argmax}} \gamma_{j_{1}, i} .
$$

We can solve the parameters $\left\{\left(\alpha_{i}, \mu_{i}, \aleph_{i}\right) \mid 1 \leq i \leq K_{1}\right\}$ as

$$
L L(D)=\ln \left(\prod_{j_{1}=1}^{m} p_{M}\left(x_{j_{1}}\left(t_{j}\right)\right)\right)=\sum_{j_{1}=1}^{m} \ln \left(\sum_{i=1}^{K_{1}} \alpha_{i} \cdot p\left(x_{j_{1}}\left(t_{j}\right) \mid \mu_{i}, \aleph_{i}\right)\right) .
$$

The expectation maximization algorithm is used for the iterative optimization solution. To maximize equation (8) by $\partial L L(D) / \partial \mu_{i}=0$, we use

$$
\sum_{j_{1}=1}^{m} \frac{\alpha_{i} \bullet p\left(x_{j_{1}}\left(t_{j}\right) \mid \mu_{i}, \aleph_{i}\right)}{\sum_{l=1}^{K 1} \alpha_{l} \bullet p\left(x_{j_{1}}\left(t_{j}\right) \mid \mu_{i}, \aleph_{i}\right)}\left(x_{j_{1}}\left(t_{j}\right)-\mu_{i}\right)=0,
$$

and with $\gamma_{j_{1}, i}=p_{M}\left(z_{j_{1}}=i \mid x_{j_{1}}\left(t_{j}\right)\right)$, we can obtain

$$
\mu_{i}=\frac{\sum_{j_{1}=1}^{m} \gamma_{j_{1},} x_{j_{1}}\left(t_{j}\right)}{\sum_{j_{1}=1}^{m} \gamma_{j_{1}, i}}
$$

where $\mu_{i}$ is the mean of each mixed component and can be estimated by the weighted average of samples. The sample weight is the posterior probability $\gamma_{j_{1}}$ of each sample belonging to the component. Similarly, from $\partial L L(D) / \partial \aleph_{i}$ $=0$, we can obtain

$$
\aleph_{i}=\frac{\sum_{j_{1}=1}^{m} \gamma_{j_{1}, i}\left(x_{j_{1}}\left(t_{j}\right)-\mu_{i}\right)\left(x_{j_{1}}\left(t_{j}\right)-\mu_{i}\right)^{T}}{\sum_{j_{1}=1}^{m} \gamma_{j_{1}, i}} .
$$

For mixed coefficient $\alpha_{i}$, in addition to maximizing $L L$ $(D)$, it needs to satisfy $\alpha_{i} \geq 0$ and $\sum_{i=1}^{K_{1}} \alpha_{i}=1$.

The Lagrange form of $L L(D)$ is

$$
L L(D)+\rho\left(\sum_{i=1}^{K_{1}} \alpha_{i}-1\right)
$$

where $\rho$ is the Lagrange multiplier. The derivative of equation (12) with respect to $\alpha_{i}$ is 0 , and

$$
\sum_{j_{1}=1}^{m} \frac{p\left(x_{j_{1}}\left(t_{j}\right) \mid \mu_{i}, \aleph_{i}\right)}{\sum_{l=1}^{K 1} \alpha_{l} \bullet p\left(x_{j_{1}}\left(t_{j}\right) \mid \mu_{l}, \aleph_{l}\right)}+\rho=0
$$

Both sides are multiplied by $\alpha_{i}$, all of the components of the mixture are summed, $\rho=-m$, and

$$
\alpha_{i}=\frac{1}{m} \sum_{j_{1}=1}^{m} \gamma_{j_{1}, i}
$$

namely, the mixing coefficient of each Gaussian component is determined by the average posterior probability of the sample.

(1) Elimination of Similar Data. According to the result of cluster set $C_{1}=\left\{C_{1,1}, C_{1,2}, C_{1,3}, \cdots, C_{1, K_{1}}\right\}$ in the second stage, the $\mathrm{CH}$ randomly weights the data generated by the nodes in the cluster with similar data simultaneously, and the TCDA algorithm is proposed to perform time-dependent deredundancy. CHs finally transmit the deredundant data $D_{C_{i, j_{1}}}\left(t_{j}\right)$ to the sink, and we have 


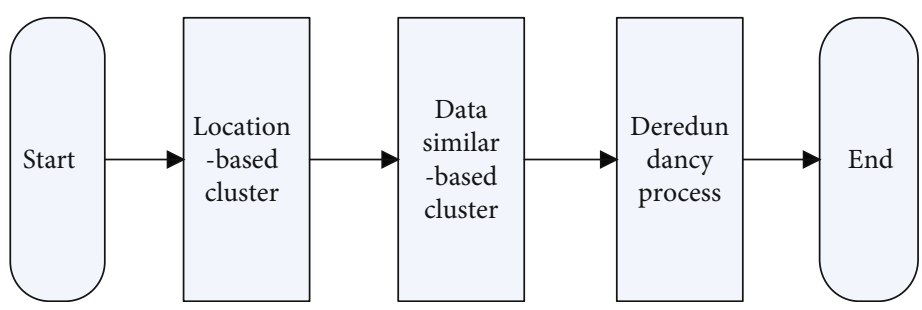

FIGURE 2: The framework of the proposed MHCSD.

Input: $L=\left\{l_{1}, l_{2}, \cdots, l_{n}\right\} ; K$

Output: Result of cluster division $C=\left\{C_{1}, C_{2}, \cdots, C_{K}\right\}$

Randomly choses $K$ sample from $L$ as initial mean vector $\left\{\mu_{1}, \mu_{2}, \cdots, \mu_{\mathrm{K}}\right\}$

Repeat

$C_{i} \longleftarrow \varnothing(1 \leq i \leq K)$

For $j \longleftarrow 1,2, \cdots, n$ do

Solving the distance $l_{j}$ and all kinds of $\mu_{i}(1 \leq i \leq K): D(i, j) \longleftarrow D_{E}(i, j)+\beta D_{P}(i, j)$

The cluster marker of $l_{j}$ is determined according to the nearest mean vector: $\tau_{j} \longleftarrow$ arg $\min _{i \in\{1,2, \cdots, K\}} D(i, j)$ $C_{\tau_{j}} \longleftarrow C_{\tau_{j}} \cup\left\{l_{j}\right\}$

End For

For $i \longleftarrow 1,2, \cdots, K$ do

$\mu_{i}^{\prime} \longleftarrow\left(1 /\left|C_{i}\right|\right) \sum_{l \in C_{i}} l$

If $\mu_{i}^{\prime} \neq \mu_{i}$ then

$\mu_{i}^{\prime} \longleftarrow \mu_{i}$

Else

Keeping the current mean vector not to change

End If

End For

Until The current mean vectors are not updated

Algorithm 1: $\operatorname{ImpkMeans}(L, K)$.

Input: $D_{C H_{h}}\left(t_{j}\right)=\left\{x_{1}\left(t_{j}\right), x_{2}\left(t_{j}\right), \cdots, x_{z}\left(t_{j}\right)\right\} ; K_{1}$

Output: Result $C_{1}=\left\{C_{1,1}, C_{1,2}, C_{1,3}, \cdots, C_{1, K_{1}}\right\}$

Repeat

For $j_{1} \longleftarrow 1,2,3, \cdots, z$ do

According to (4.7) calculating the posterior probability generated by the mixed components $x_{j_{1}}\left(t_{j}\right)$,

$\gamma_{j_{1}, i} \longleftarrow p_{M}\left(z_{j_{1}}=i \mid x_{j_{1}}\left(t_{j}\right)\right)\left(1 \leq i \leq K_{1}\right)$

End For

For $i \longleftarrow 1,2,3, \cdots, K_{1}$ do

$$
\begin{aligned}
& \mu_{i}{ }^{\prime} \longleftarrow\left(\sum_{j_{1}=1}^{m} \gamma_{j_{1}, i} x_{j_{1}}\left(t_{j}\right) / \sum_{j_{1}=1}^{m} \gamma_{j_{1}, i}\right) \\
& \aleph_{i}{ }^{\prime} \longleftarrow\left(\sum_{j_{1}=1}^{m} \gamma_{j_{1}, i}\left(x_{j_{1}}\left(t_{j}\right)-\mu_{i}{ }^{\prime}\right)\left(x_{j_{1}}\left(t_{j}\right)-\mu_{i}{ }^{\prime}\right)^{T} / \sum_{j_{1}=1}^{m} \gamma_{j_{1}, i}\right) \\
& \alpha_{i}{ }^{\prime} \longleftarrow(1 / m) \sum_{j_{1}=1}^{m} \gamma_{j_{1}, i}
\end{aligned}
$$

\section{End For}

Update model parameters: $\left\{\left(\alpha_{i}, \mu_{i}, \aleph_{i}\right) \mid 1 \leq i \leq K_{1}\right\} \longleftarrow\left\{\left(\alpha_{i}{ }^{\prime}, \mu_{i}{ }^{\prime}, \aleph_{i}{ }^{\prime}\right) \mid 1 \leq i \leq K_{1}\right\}$

Until Satisfy the stop condition

$C_{i} \neq \varnothing\left(1 \leq i \leq K_{1}\right)$

For $j_{1} \longleftarrow 1,2,3, \cdots, z$ do

According to (4.7) determining cluster markets $\rho_{j_{1}}$ of $x_{j_{1}}\left(t_{j}\right)$

$C_{1, \rho_{j_{1}}} \longleftarrow C_{1, \rho_{j_{1}}} \cup\left\{x_{j_{1}}\left(t_{j}\right)\right\}$

End For

Algorithm 2: $\operatorname{GMM}\left(D_{C_{h}}\left(t_{j}\right), K_{1}\right)$. 
Input: $L ; K ; D_{\mathrm{CH}_{h}}\left(t_{j}\right) ; K_{1}$

Output: Deredundancy data

Resut1 $=\operatorname{ImpkMeans}(L, K)$

Rerult2 $=\mathrm{GMM}\left(D_{\mathrm{CH}_{h}}\left(t_{\mathrm{j}}\right), K_{1}\right)$

Result3 is that $\mathrm{CH}$ randomly weights the data generated by the nodes in the cluster with similar data.

TCDA algorithm will process Result3 data.

Algorithm 3: $\operatorname{HMDA}\left(L, K, D_{\mathrm{CH}_{h}}\left(t_{j}\right), K_{1}\right)$.

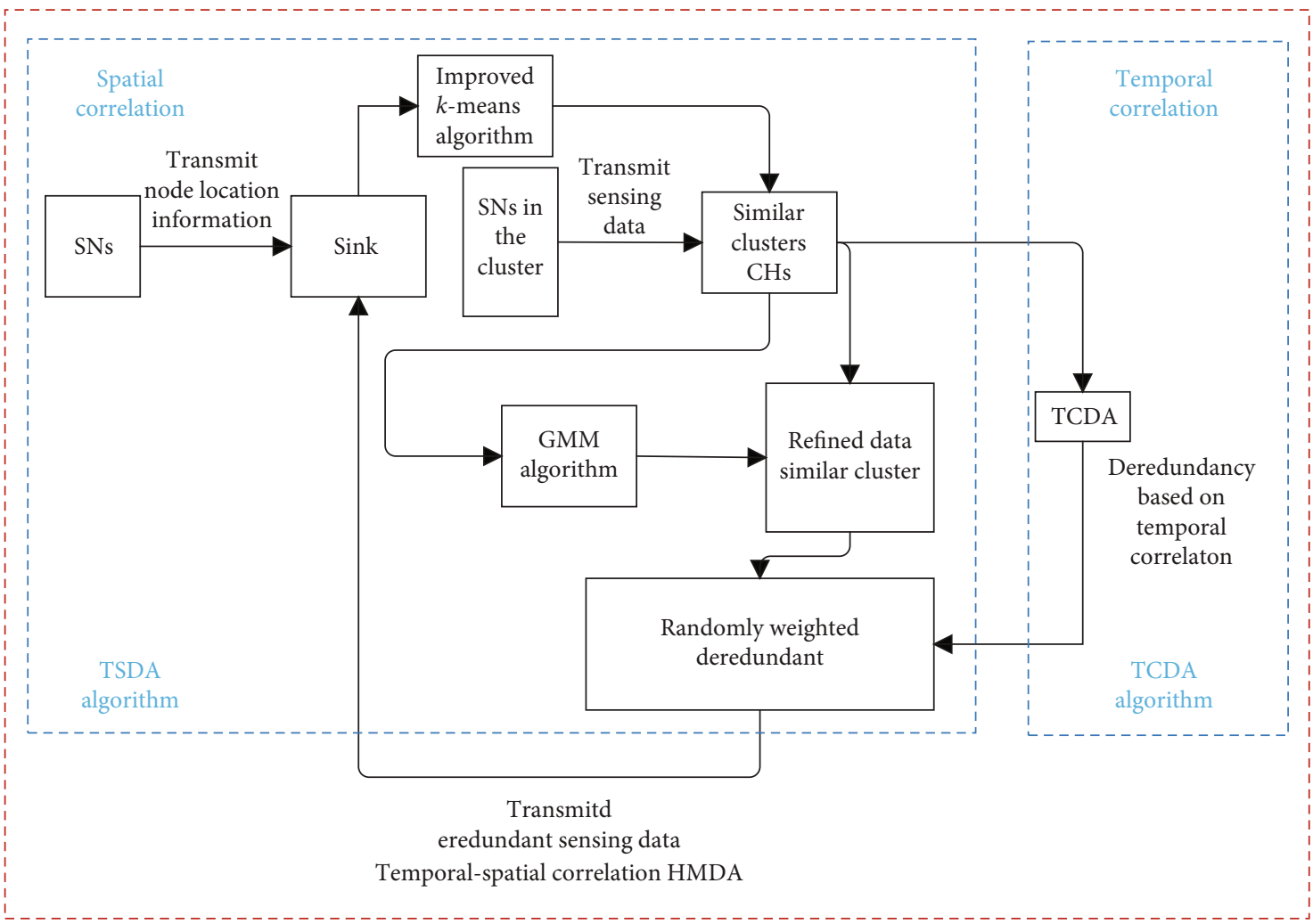

FIgURE 3: The flow chart of HMDA.

$$
D_{C_{i, j}}\left(t_{j}\right)=\beta_{1} x_{w}\left(t_{j}\right)+\beta_{2} x_{a}\left(t_{j}\right)+\cdots+\beta_{v} x_{b}\left(t_{j}\right),
$$

where $\beta_{1}, \beta_{2}, \cdots, \beta_{v}$ are weighting factors; $\sum_{j=1}^{v} \beta_{j}=1 ; x_{w}\left(t_{j}\right)$, $x_{a}\left(t_{j}\right), \cdots, x_{b}\left(t_{j}\right)$ are the sensing data generated from nodes $s_{w}, s_{a}, \cdots, s_{b}$ at $t_{j}$; and $s_{w}, s_{a}, \cdots, s_{b} \in C_{i j_{1}} \& \& 0<w, a, \cdots, b \leq n$ $\& \& 0<i \leq K \& \& 0<j_{1} \leq K_{1}$.

3.2.3. HMDA Algorithm. To reduce redundant data in WSNs, a hybrid multistage deredundancy algorithm (HMDA), which combines MHCSD and TCDA to reduce redundant data comprehensively, is proposed based on spatialtemporal correlations, as shown in Algorithm 3.

The MHCSD algorithm reduces redundancy in terms of spatial correlations, and the TCDA algorithm further reduces redundant data in terms of temporal correlations. TCDA fully considers the following factors in the process of deduplication: when the range of data variation is large, there is a large error in the local maximum or minimum value and a missing local eigenvalue, and when the data fluctuation is stable, the data similarity threshold cannot work effectively. Considering the ratio of deduplication, TCDA guarantees the timeliness of the sensing data with a maximum time threshold to prevent a failure in the data similarity threshold. Furthermore, an adaptive step size mechanism is proposed to reduce the complexity of calculation and energy consumption. Hence, HMDA reduces network energy consumption and extends the lifetime of a WSN simultaneously. In addition, the flow chart of HMDA is shown in Figure 3.

\subsection{Performance Analysis}

3.3.1. Algorithm Complexity. In the first stage, the sink aggregates through all node positions, classifies all nodes, and assumes that model training requires $f_{1}$ cycles. In the first step, the position set and classification number $K$ of $n$ nodes are input, and the time complexity is $O(n+1)$; in the second step, $K$ samples are randomly selected as the initial mean 
vector, and the time complexity is $O(K)$; in the third step, the distance between each sample and $K$ means is calculated, and the time complexity is $O\left(f_{1} \times n \times K\right)$; in the fourth step, the mean vector is updated, and the time complexity is $O\left(f_{1} \times\right.$ $K \times q) \& \&(1<q<n)$; in the fifth step, the cluster division results are output, and the time complexity is $O(K \times q) \& \&$ $(1<q<n)$. In the second stage, the $\mathrm{CH}$ performs a data similarity analysis of the cluster data generated at each moment, assuming that model training requires $f_{2}$ cycles. The first step is to input the sensing data of $z$ nodes and similarity number $K_{1}$, and the time complexity is $O(z+1)$; the second step is to calculate the posterior probability generated by each mixed component, and the time complexity is $O$ $\left(f_{2} \times z\right)$; the third step is to calculate each model parameter, and the time complexity is $O\left(f_{2} \times K_{1}\right)$; the fourth step is to calculate the cluster tag's classification, and the calculation complexity is $O\left(z \times K_{1}\right)$; the fifth step is to output $K_{1}$ classification clusters, and the time complexity is $O\left(z \times K_{1}\right)$. In the third stage, the $\mathrm{CHs}$ perform random weighted transmission to reduce redundant data in similar nodes, and the time complexity is $O\left(z \times K_{1}\right)$.

Hence, we can obtain that the complexity of the model scheme is $\max \left(O\left(f_{1} \times n \times K\right), O\left(f_{1} \times K \times q\right), O\left(f_{2}\right.\right.$ $\left.\times z), O\left(f_{2} \times K_{1}\right)\right)$.

3.3.2. Energy Consumption. Most of the energy in the sensor node is consumed by its transceiver module. The channel model of the transmitter has two kinds of free space models and multipath fading models, and the energy consumption is related not only to the amount of data but also to the transmission distance $d$. Therefore, the energy consumption $E_{T X}$ $(N, d)$ of the node to send $N$-bit data is

$$
E_{T X}(N, d)=\left\{\begin{array}{l}
N \times E_{\text {elec }}+N \times \varepsilon_{m p} \times d^{4}, d>d_{0}, \\
N \times E_{\text {elec }}+N \times \varepsilon_{f s} \times d^{2}, d \leq d_{0},
\end{array}\right.
$$

where $E_{\text {elec }}$ represents the energy consumption of the circuit sending or receiving data and $\varepsilon_{m p}$ and $\varepsilon_{f s}$, respectively, represent the energy consumption of the signal amplifier:

$$
d_{0}=\left(\frac{\varepsilon_{f s}}{\varepsilon_{m p}}\right)^{1 / 2}
$$

The energy consumption $E_{R X}(N)$ of the node receiving $N$-bit data is

$$
E_{R X}(N)=N \times E_{\text {elec }}
$$
data is

The energy consumption of nodes processing $N$-bit

$$
E_{P}(N)=N \times E_{P}
$$

where $E_{P}$ represents the energy consumption of processing unit data. The node's remaining energy consumption $E_{r}$ is

$$
E_{r}=E_{0}-\left(E_{T X}\left(N_{1}, d\right)+E_{R X}\left(N_{2}\right)+E_{P}\left(N_{3}\right)\right)
$$

where $E_{r}$ represents the remaining energy consumption of the node, $E_{0}$ represents the initial energy, $N_{1}$ represents the total amount of data transmitted, $N_{2}$ represents the total amount of received data, and $N_{3}$ represents the total amount of data processing.

\section{Experimental Results}

4.1. Experimental Setup. To verify the effectiveness of the proposed method, the temperature sensing data from the Intel Berkeley Laboratory are used [35]; these data include 54 nodes, and each node collects sensing data every 0.5 minutes. The map is shown in Figure 4 . To verify the deredundancy ratio of edge sensing data and the network lifetime, the data transmission model and node energy consumption model are adopted. The experiments consider the following metrics: the deredundancy ratio, the deredundancy error, the influence of the amount of similar data clusters $K_{1}$ on the deredundancy ratio, and the energy consumption. The proposed HMDA will be compared with the TCDA, TSDA, and Dat algorithms [9]. The parameters and their values are shown in Table 2.

4.2. Performance Evaluation. First, the performance results of the three stages are analyzed separately. In the first stage, clustering classification positions of similar nodes are obtained; in the second stage, clustering classification of similar data nodes is obtained; in the third stage, as a result of the first and second stages, the generated sensing data are made deredundant by means of random weighting. Second, the influence of $K_{1}$ on the deredundancy ratio in the second stage is analyzed. Finally, the energy consumption is analyzed with Dat [9], TCDA, TSDA, and HMDA.

In the first stage, the sink performs clustering according to the nodes' coordinate positions by running the improved $k$-means clustering algorithm. $K$ is assumed to be 4 , and $\beta=\{0,0.3,0.5,0.7,1\}$. The results of the four clusters also change significantly as $\beta$ varies. The diamond in the figure represents the cluster center of the four clusters. The node's cluster distribution probability is shown in Table 3, and the clustering results are shown in Figure 5.

According to the probability ratio, the sink classifies the nodes that are prone to change into corresponding clusters. As shown in Table 3 , the classification results are $C_{1}=$ $\{0,2,21,22,23,24,25,26,27,28,29,30,31,32,33\}, C_{2}=\{1$ , 34, 35, 36, 37, 38, 39, 40, 41, 42, 43, 44\}, $C_{3}=\{3,4,5,6,7,8$, $9,45,46,47,48,49,50,51,52,53\}$, and $C_{4}=\{10,11,12,13$, $14,15,16,17,18,19,20\}$.

In the second stage, the $\mathrm{CHs}$ perform Gaussian mixture clustering. By successively acquiring edge sensing data from nodes within each cluster, the $\mathrm{CH}$ s can analyze data similarity according to further improve the deredundancy ratio. Similar classification results in cluster $C_{1}$ are shown in Figure 6. 


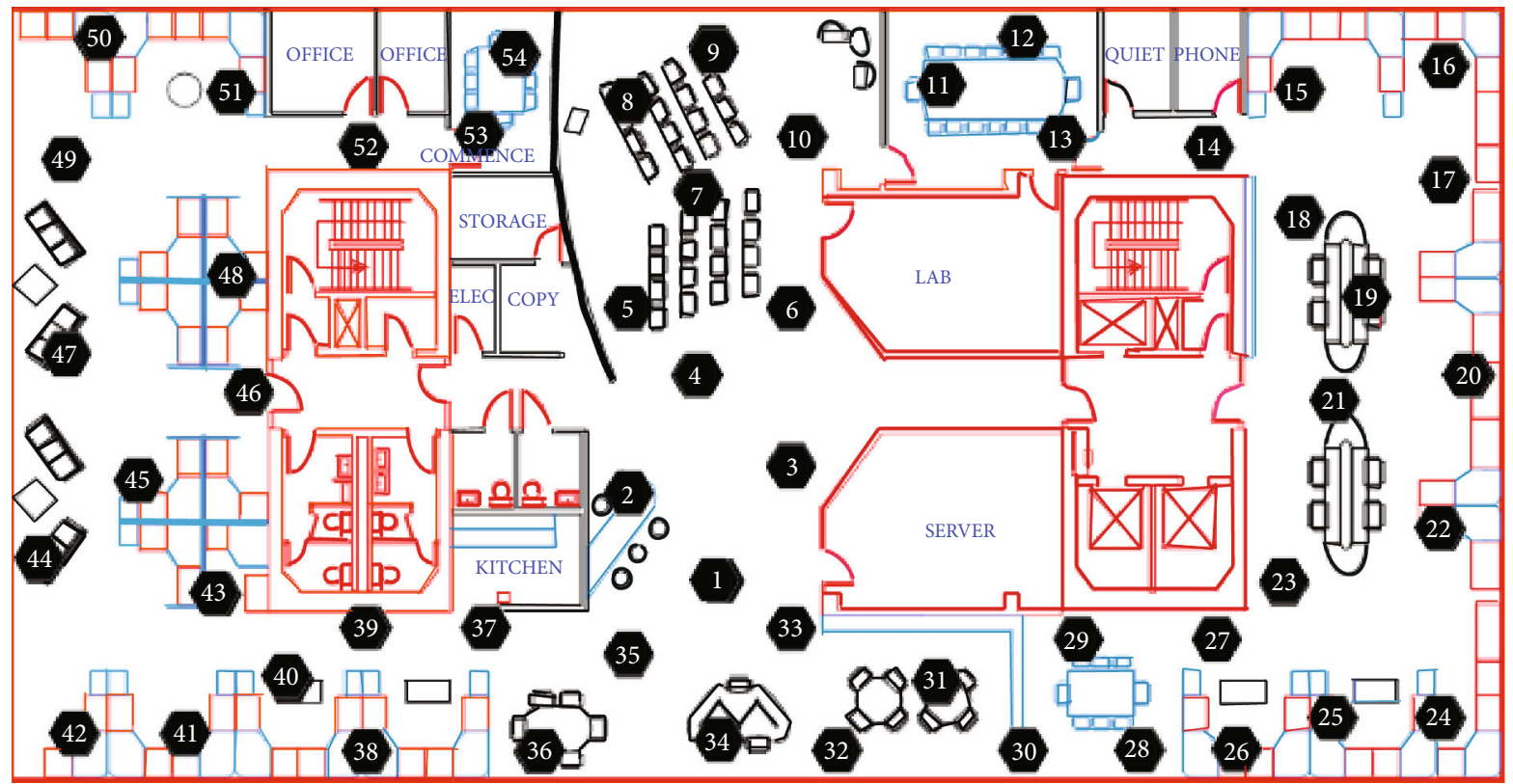

FIgURE 4: The map of sensor nodes in the experiment.

TABLE 2: Parameters of the experiments.

\begin{tabular}{lc}
\hline Parameter & Value \\
\hline Duration & Feb. $28 \sim$ Apr. 5, \\
& 2004 \\
Area coverage & $42 \mathrm{~m} \times 33 \mathrm{~m}$ \\
Observation parameter & Temperature \\
Node size of WSNs & 54 \\
The number of perceived data collected/ten & 230 \\
thousand & 31 \\
Collection interval $(\mathrm{s})$ & 10 \\
Distance of CHs and sink $(\mathrm{m})$ & 50 \\
$E_{\text {elec }}(\mathrm{nJ} / \mathrm{bit})$ & 0.0013 \\
$\varepsilon_{m p}\left(\mathrm{pJ} / \mathrm{bit} / \mathrm{m}^{4}\right)$ & 100 \\
$\varepsilon_{f s}\left(\mathrm{pJ} / \mathrm{bit} / \mathrm{m}^{2}\right)$ & 5 \\
$E_{P}(\mathrm{~nJ} / \mathrm{bit})$ & 5 \\
$E_{0}(\mathrm{~J})$ & \\
\hline
\end{tabular}

As shown in Figure 6, $C_{1}$ is divided into 4 subclusters: $C_{11}=\{22,25,28,30,32\}, C_{12}=\{23,24,26\}, C_{13}=\{27,29$, $31,33\}$, and $C_{14}=\{0,2,21\}$. Similarly, cluster $C_{2}$ includes 3 subclusters: $C_{21}=\{1,34,35,36\}, C_{22}=\{37,38,39\}$, and $C_{23}$ $=\{40,41,43,44\} ; C_{3}$ is classified into $C_{31}=\{3,4,5,6,7\}$, $C_{32}=\{8,9,45,46\}$, and $C_{33}=\{47,48,49,50,51,52,53\}$; and $C_{4}$ is divided into $C_{41}=\{10,11,12\}, C_{42}=\{13,14,15,16\}$, and $C_{43}=\{17,18,19,20\}$.

In the third stage, the data in similar clusters will be randomly weighted to optimize the redundancy ratio. For subcluster $C_{13}=\{27,29,31,33\}$ in cluster $C_{1}$, the deredundancy performance, redundancy error, and mean square error are shown in Figures 7 and 8 and Table 4, respectively.
TABLE 3: Node distribution probability in clusters.

\begin{tabular}{lcccc}
\hline Node ID & Cluster 1 & Cluster 2 & Cluster 3 & Cluster 4 \\
\hline 0 & $60 \%$ & $40 \%$ & 0 & 0 \\
2 & $60 \%$ & $40 \%$ & 0 & 0 \\
5 & 0 & 0 & $80 \%$ & $20 \%$ \\
9 & 0 & 0 & $80 \%$ & $20 \%$ \\
10 & 0 & 0 & $40 \%$ & $60 \%$ \\
19 & $20 \%$ & 0 & 0 & $80 \%$ \\
20 & $20 \%$ & 0 & 0 & $80 \%$ \\
32 & $60 \%$ & $40 \%$ & 0 & 0 \\
33 & $60 \%$ & $40 \%$ & 0 & 0 \\
45 & 0 & $40 \%$ & $60 \%$ & 0 \\
46 & 0 & $40 \%$ & $60 \%$ & 0 \\
\hline
\end{tabular}

As shown in Figure 7, the sensing data of subcluster $C_{13}$ tend to be the middle values with randomly weighted optimization. The sensing data of node 29 and node 33 are close to the deredundancy results. However, the values of node 27 and node 31 are relatively far away. From Figure 8, we can see that the mean square errors of nodes 29 and 33 are relatively lower than those of nodes 27 and 31. According to the results in Table 4, it can be seen that the mean square errors of nodes 27, 29, 31, and 33 are 0.035, 0.004, 0.034, and 0.006 , respectively, which indicates that even if the data are similar, there are still differences between the sensing data. Therefore, for the methods of data similarity analysis with the coordinates of nodes, the lack of spatial correlation analysis can cause greater errors. Multistage clustering improves the accuracy of sensing data similarity.

Since the deredundancy ratio of MHCSD is related to the data similarity clustering $K_{1}$ in the second stage and the value 


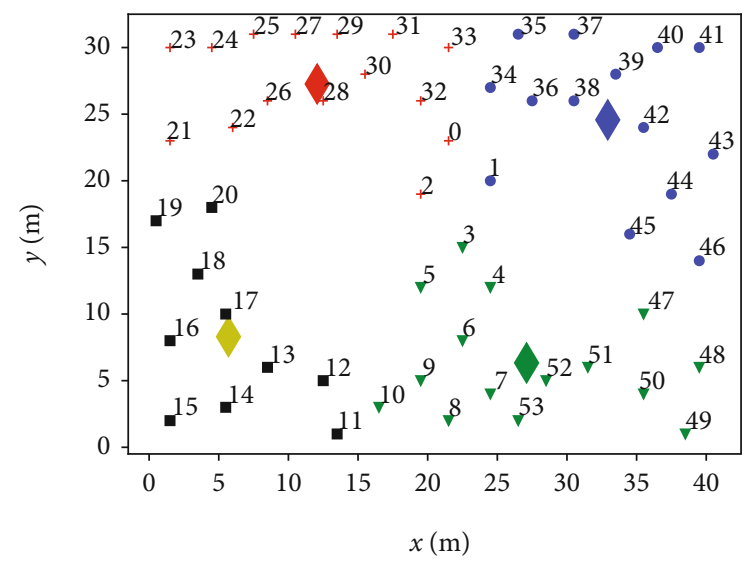

(a) $\beta=0$

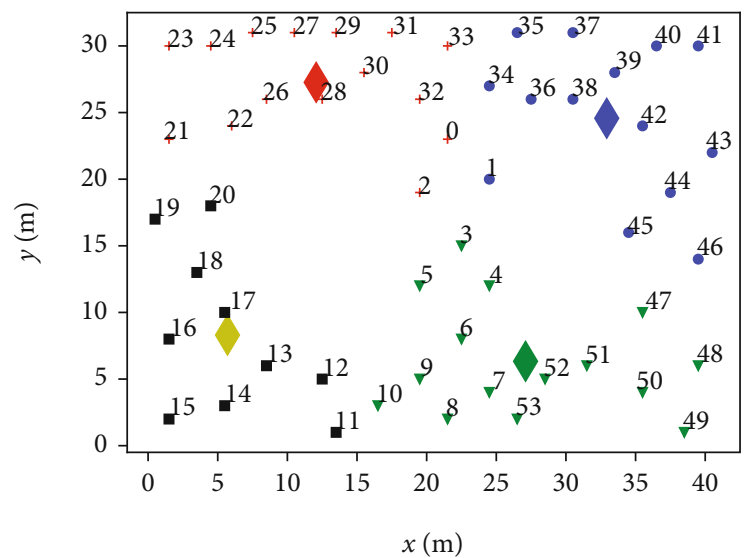

(c) $\beta=0.5$

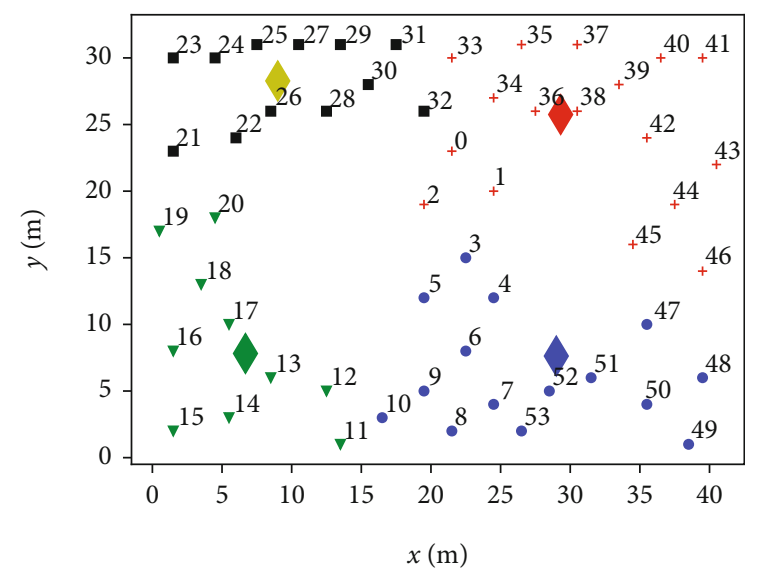

(b) $\beta=0.3$

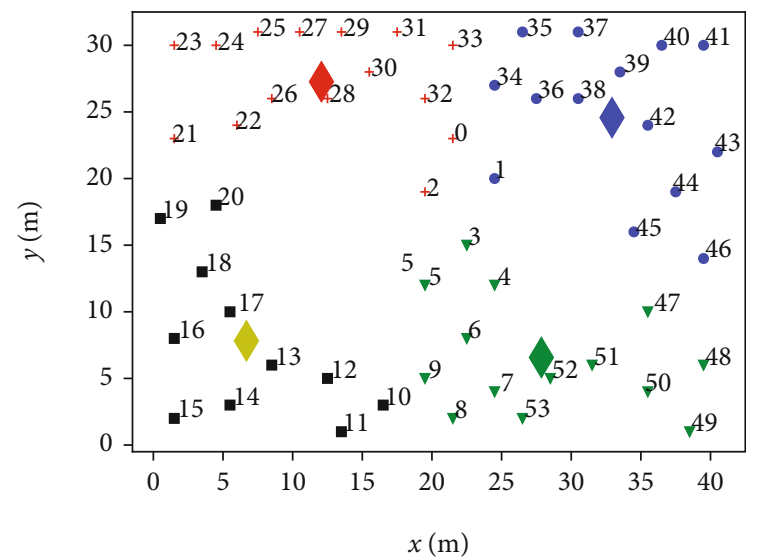

(d) $\beta=0.7$

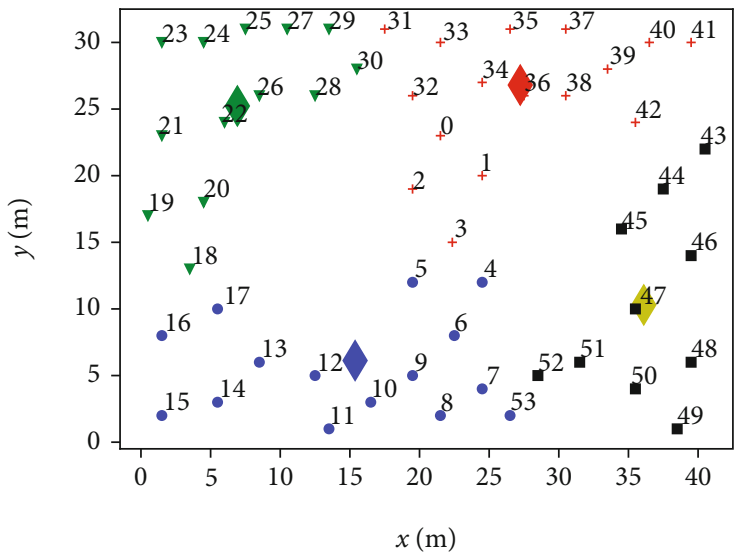

(e) $\beta=1$

Figure 5: Node clustering of the improved $k$-means scheme with varying $\beta$.

of $K_{1}$ affects the accuracy of the data correlation, the effect of $K_{1}$ on the deredundancy ratio is shown in Figure 9.

As shown in Figure 9, the deredundancy ratio gradually decreases as $K_{1}$ increases. When $K_{1}=1$, it indicates that clusters $C_{1}, C_{2}, C_{3}$, and $C_{4}$ are not divided into any similar subclusters, and MHCSD treats all nodes in clusters $C_{1}, C_{2}, C_{3}$, and $C_{4}$ as redundant nodes, which perform random weighting to optimize sensing data. Therefore, the deredundancy ratio is maximized. However, when $K_{1}=1$, it is equivalent to clustering all nodes by position similarity without considering the data similarity cluster, which leads to a larger error. When $K_{1}=10$, MHCSD classifies the nodes into 10 similar subdata clusters in each cluster $C_{1}, C_{2}, C_{3}$, and $C_{4}$ and randomly weights them to deduplicate the sensing data. Hence, the redundancy ratio is the lowest, which guarantees the accuracy of the deredundant data. To ensure both the 


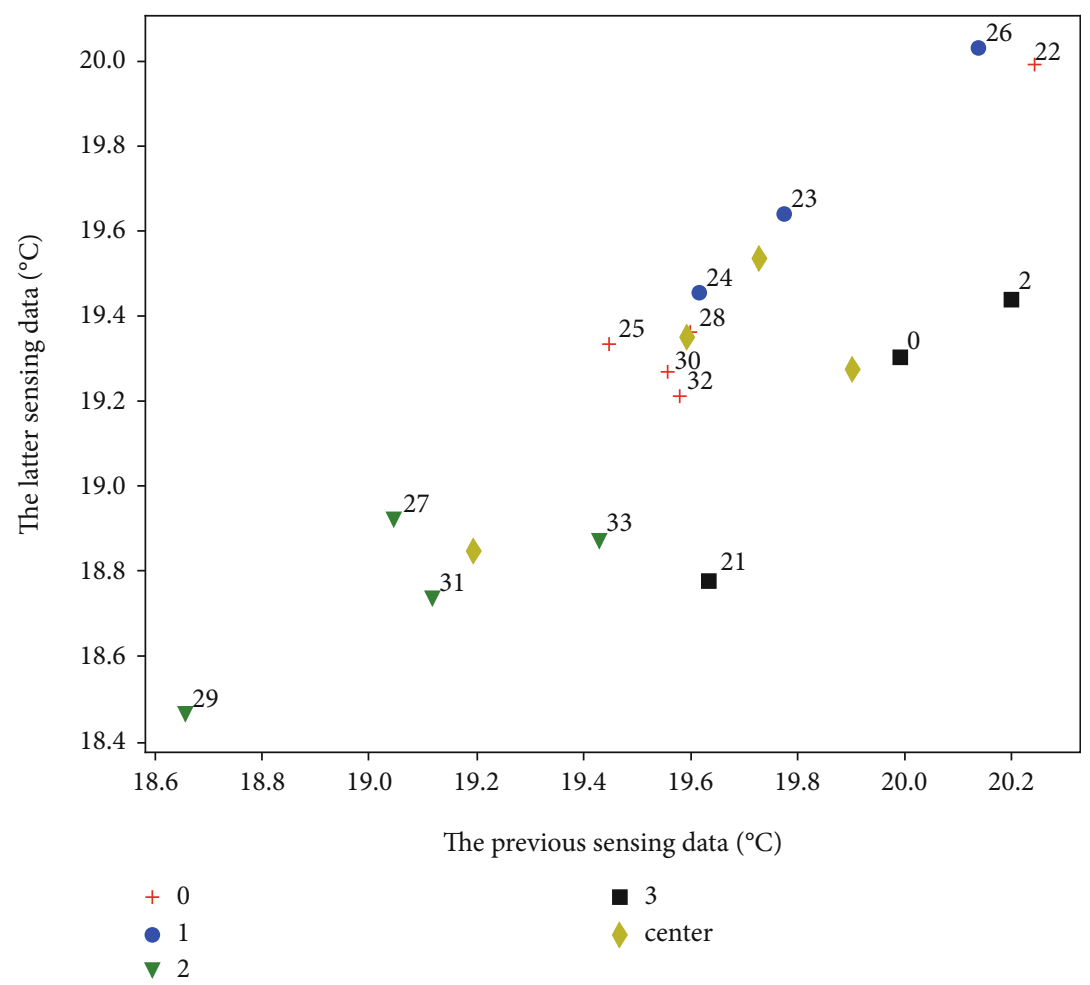

Figure 6: Data similarity distribution of $C_{1}$.

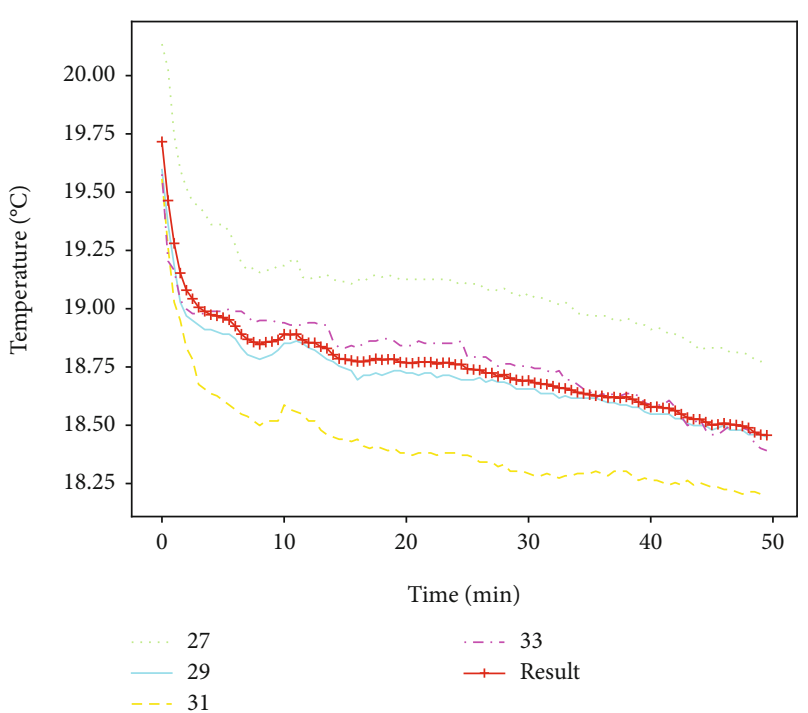

Figure 7: Deredundancy result of $C_{13}$.

accuracy of the data and the deredundancy ratio of the data, we set $K_{1}=4$ in the following performance analysis.

Figure 10 shows that as the number of nodes increases, the deredundancy ratio also increases and varies between $65 \%$ and $75 \%$. When the number of nodes is 23 , the deredundancy ratio is the highest (75\%). When the number of nodes is less than 3 , the proposed scheme omits the spatial correlation deredundancy and transmits the sensing data to the corresponding $\mathrm{CHs}$, which degrades the deredundancy ratio.

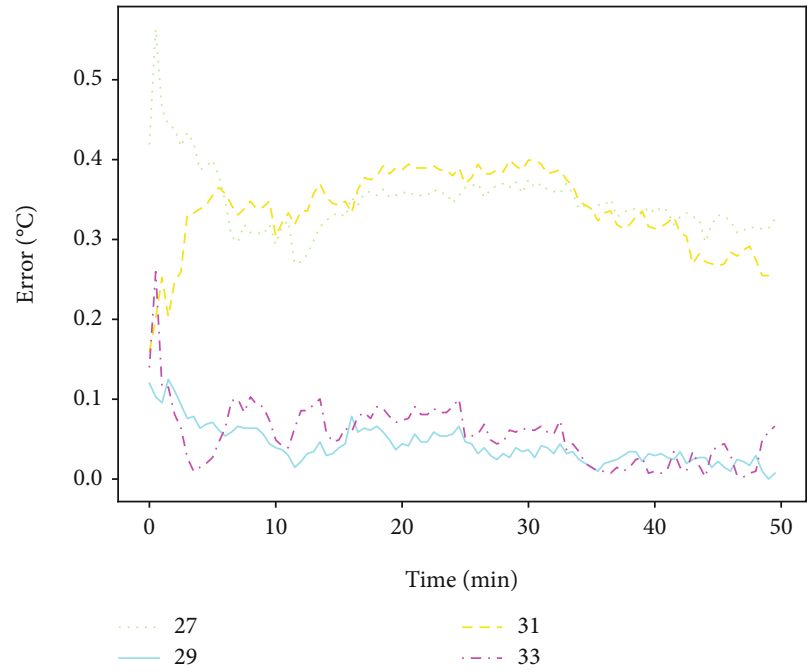

FIgURE 8: Deredundancy error of $C_{13}$.

TABLE 4: The mean square errors of nodes in $C_{13}$.

\begin{tabular}{lc}
\hline Node & Mean square error \\
\hline 27 & 0.035 \\
29 & 0.004 \\
31 & 0.034 \\
33 & 0.006 \\
\hline
\end{tabular}




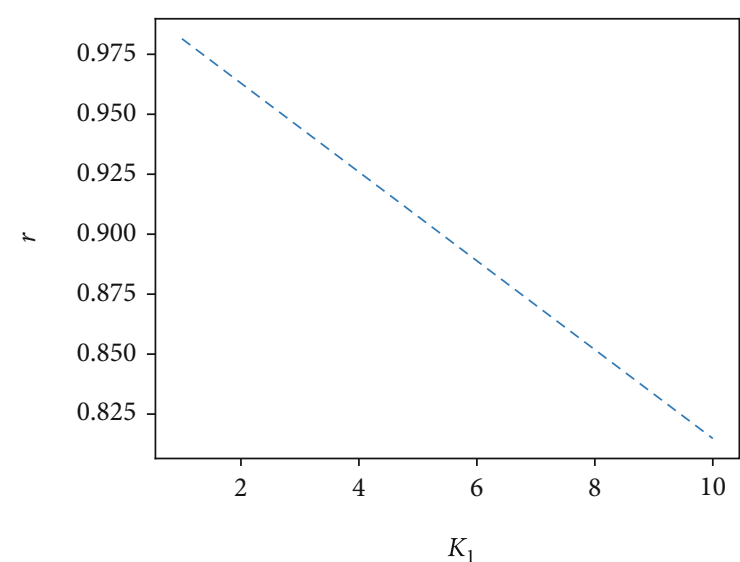

FIGURE 9: Effect of $K_{1}$ on deredundancy ratio.

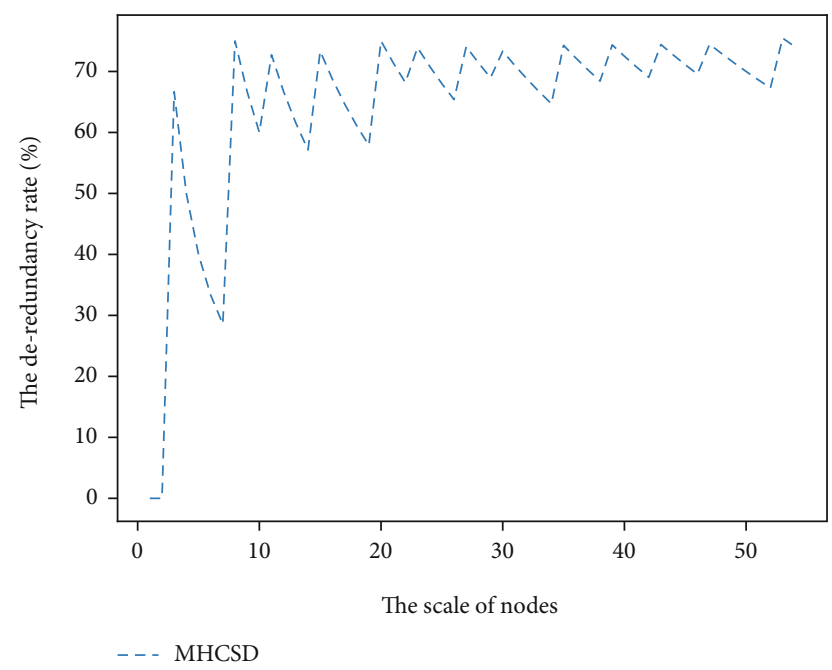

FIgURE 10: The deredundancy ratio of MHCSD.

When the number of nodes is larger than 3, MHCSD performs a spatial correlation deredundancy algorithm, deduplicates redundant nodes in clusters, and obviously improves the deredundancy ratio.

As seen from Figure 11, the deredundancy ratio of the HMDA algorithm varies between $97.50 \%$ and $98.0 \%$, which is obviously higher than those of TCDA and Dat. Compared with TCDA and Dat, the deredundancy ratio of HMDA increases by $1.7 \%$ and $4.7 \%$, respectively. Therefore, HMDA combines MHCSD and TCDA to reduce redundant data comprehensively and can further remove $70 \%$ of the redundant data. Additionally, the accuracy of the deredundancy nodes is maintained between 0.004 and 0.035 , and within the allowable error range for a user, the deredundancy ratio reaches the highest. The results in Figure 11 also verify that HMDA is effective in improving the deredundancy ratio based on spatial-temporal correlations.

The energy consumed by different schemes is shown in Figure 12. The energy consumed by the four algorithms increases gradually as the number of nodes increases. Among the different schemes, the energy consumption of HMDA is much lower than those of the other three algorithms. For

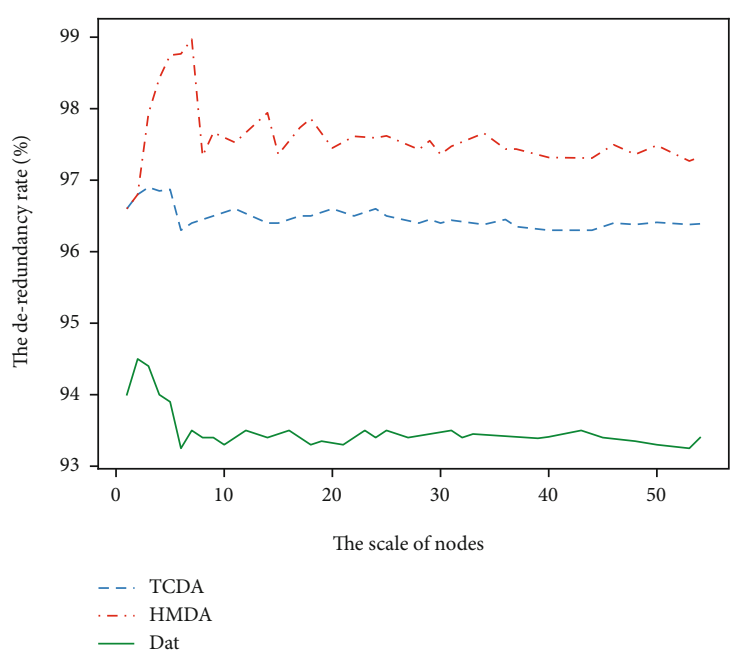

FIgURE 11: Deredundancy ratio of different schemes.

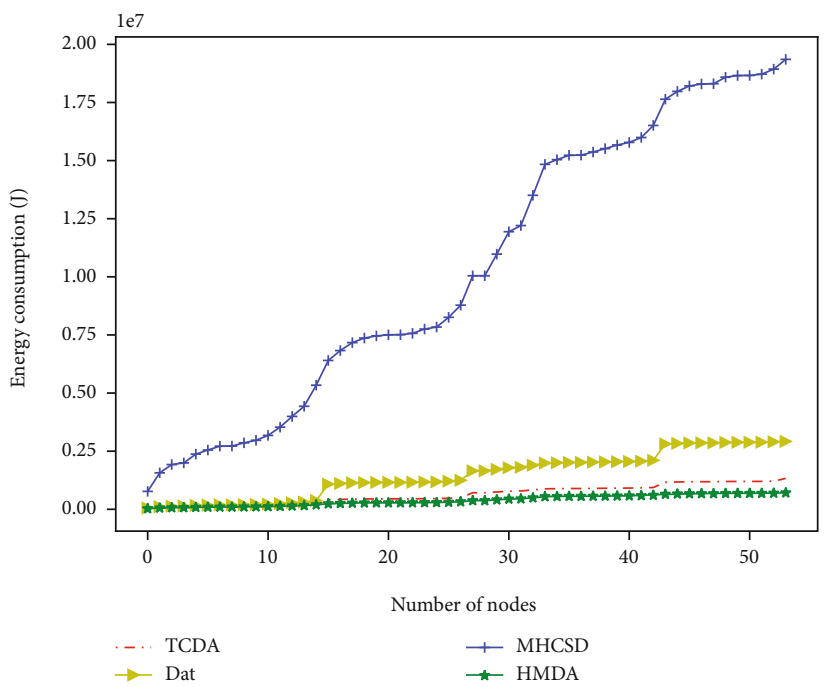

FIGURE 12: Energy consumptions of different schemes.

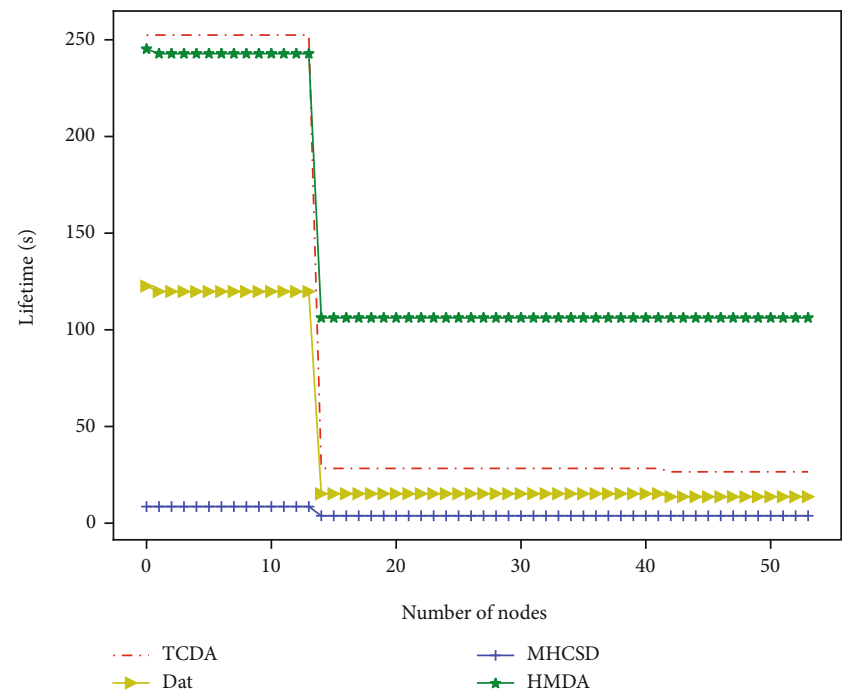

FIgURE 13: Lifetimes of different schemes. 
the proposed HMDA scheme, energy consumption increases very slowly. When the number of nodes is 50 , the energy consumption of HMDA is only $0.12 \mathrm{~J}$, which is obviously lower than those of the other three algorithms. The reason is that HMDA will adaptively perform both spatial correlation and temporal correlation analyses.

The network lifetimes of different schemes are shown in Figure 13. When the number of nodes is lower than 15 , the lifetimes of HMDA, TCDA, Dat, and MHCSD remain stable at $260 \mathrm{~s}, 250 \mathrm{~s}, 120 \mathrm{~s}$, and $15 \mathrm{~s}$, respectively. The reason is that the deredundancy ratios of the 4 schemes are $97.5 \%, 96.3 \%$, $93 \%$, and $70 \%$, which ensures that all nodes perform the same data processing scheme with constant energy consumption. It is obvious that the lifetime of HMDA is longer than that of the other 3 schemes. Especially when the number of nodes increases to 50, the lifetime of HMDA is 109 s, which is 12.6, 3.0, and 3.9 times higher than those of MHCSD, TCDA, and Dat, respectively. The results in Figures 11-13 demonstrate that the proposed HMDA scheme can achieve better performance in terms of the deredundant ratio, energy consumption, and network lifetime.

\section{Conclusion}

Focusing on the problem of data redundancy in WSNs, a multistage hierarchical clustering deredundancy algorithm is proposed to decrease the additional power consumption and extend the lifetime of a WSN. Based on the improved $k$ -means clustering method, all nodes are classified according to the node position information and temporal similarity. The Gaussian hybrid clustering method is adopted to improve the redundant similarity of edge nodes. According to the secondary classification results, the sensing data generated by the redundant nodes are randomly weighted to remove the redundant data. Detailed analysis and experimental results show that, compared with the existing schemes, the proposed scheme is superior in terms of the deredundancy ratio, power consumption, and lifetime of a WSN.

\section{Data Availability}

The data used to support the findings of this study are available from the corresponding author upon reasonable request.

\section{Conflicts of Interest}

The authors declare that there are no conflicts of interest regarding the publication of this paper.

\section{Acknowledgments}

This work is supported by the National Science Foundation of China (No. 61772562, 62062019), the Key Project of Hubei Provincial Science and Technology Innovation Foundation of China (No. 2018ABB1485), the Hubei Provincial Natural Science Foundation of China (No. 2019CFB815), the Fundamental Research Funds for the Central Universities (No. CZP19004), and the Youth Elite Project of State Ethnic Affairs Commission of China (No. 2016-3-08).

\section{References}

[1] P. Zeng, B. Pan, K.-K. R. Choo, and H. Liu, "MMDA: multidimensional and multidirectional data aggregation for edge computing-enhanced IoT," Journal of Systems Architecture, vol. 106, article 101713, 2020.

[2] S. Wan, X. Li, Y. Xue, W. Lin, and X. Xu, "Efficient computation offloading for internet of vehicles in edge computingassisted 5G networks," The Journal of Supercomputing, vol. 76, no. 4, pp. 2518-2547, 2020.

[3] F. Ud Din, A. Ahmad, H. Ullah, A. Khan, T. Umer, and S. Wan, "Efficient sizing and placement of distributed generators in cyber-physical power systems," Journal of Systems Architecture, vol. 97, pp. 197-207, 2019.

[4] S. Wan, L. Qi, X. Xu, C. Tong, and Z. Gu, "Deep learning models for real-time human activity recognition with smartphones," Mobile Networks and Applications, vol. 25, no. 2, pp. 743-755, 2020

[5] W. Tang, X. Zhao, W. Rafique, L. Qi, W. Dou, and Q. Ni, “An offloading method using decentralized P2P-enabled mobile edge servers in edge computing," Journal of Systems Architecture, vol. 94, pp. 1-13, 2019.

[6] S. Wan, Z. Gu, and Q. Ni, "Cognitive computing and wireless communications on the edge for healthcare service robots," Computer Communications, vol. 149, pp. 99-106, 2020.

[7] G. B. Tayeh, A. Makhoul, C. Perera, and J. Demerjian, “A spatial-temporal correlation approach for data reduction in cluster-based sensor networks," IEEE Access, vol. 7, pp. 50669-50680, 2019.

[8] A. K. Idrees, A. K. M. Al-Qurabat, C. A. Jaoude, and W. L. Al-Yaseen, "Integrated divide and conquer with enhanced $\mathrm{k}$-means technique for energy-saving data aggregation in wireless sensor networks," in 2019 15th International Wireless Communications \& Mobile Computing Conference (IWCMC), pp. 973-978, Tangier, Morocco, June 2019.

[9] R. Alhussaini, A. K. Idrees, and M. A. Salman, "Data transmission protocol for reducing the energy consumption in wireless sensor networks," in International Conference on New Trends in Information and Communications Technology Applications, pp. 35-49, Baghdad, Iraq, 2018.

[10] S. Chowdhury, A. Roy, A. Benslimane, and C. Giri, "On semantic clustering and adaptive robust regression based energy-aware communication with true outliers detection in WSN," Ad Hoc Networks, vol. 94, article 101934, 2019.

[11] Y. Fathy, P. Barnaghi, and R. Tafazolli, "An adaptive method for data reduction in the internet of things," in 2018 IEEE 4th World Forum on Internet of Things (WF-IoT), pp. 729735, Singapore, February 2018.

[12] W. M. Elsayed, H. M. El-Bakry, and S. M. EL-Sayed, "Data reduction using integrated adaptive filters for energy-efficient in the clusters of wireless sensor networks," IEEE Embedded Systems Letters, vol. 11, no. 4, pp. 119-122, 2019.

[13] N. A. M. Alduais, J. Abdullah, A. Jamil, and H. Heidari, "Performance evaluation of real-time multivariate data reduction models for adaptive-threshold in wireless sensor networks," IEEE Sensors Letters, vol. 1, no. 6, pp. 1-4, 2017.

[14] L. Tan and M. Wu, "Data reduction in wireless sensor networks: a hierarchical LMS prediction approach," IEEE Sensors Journal, vol. 16, no. 6, pp. 1708-1715, 2016.

[15] N. R. Roy and P. Chandra, "Analysis of data aggregation techniques in WSN," International Conference on Innovative Computing and Communications, vol. 2, pp. 571-581, 2020. 
[16] D. Yuvaraj, M. Sivaram, and S. Navaneetha Krishnan, "Intelligent detection of untrusted data transmission to optimize energy in sensor networks," Journal of Information and Optimization Sciences, vol. 41, no. 3, pp. 799-811, 2020.

[17] R. Banerjee, S. Chatterjee, and S. Das Bit, "Performance of a partial discrete wavelet transform based path merging compression technique for wireless multimedia sensor networks," Wireless Personal Communications, vol. 104, no. 1, pp. 5771, 2019.

[18] S. Diwakaran, B. Perumal, and K. Vimala Devi, "A cluster prediction model-based data collection for energy efficient wireless sensor network," The Journal of Supercomputing, vol. 75, no. 6, pp. 3302-3316, 2019.

[19] C. Xu, K. Wang, Y. Sun, S. Guo, and A. Y. Zomaya, "Redundancy avoidance for big data in data centers: a conventional neural network approach," IEEE Transactions on Network Science and Engineering, vol. 7, no. 1, pp. 104-114, 2020.

[20] S. Kumar and V. K. Chaurasiya, "A strategy for elimination of data redundancy in Internet of things (IoT) based wireless sensor network (WSN)," IEEE Systems Journal, vol. 13, no. 2, pp. 1650-1657, 2019.

[21] J. Zhang, H. Huang, Y. Luo, Y. Fan, and G. Yang, "Immunization-based redundancy elimination in mobile opportunistic networks- generated big data," Future Generation Computer Systems, vol. 79, pp. 920-927, 2018.

[22] Y. Li, Z. Lv, J. Zhao, and Z. Pan, "Improving performance of medical image fusion using histogram, dictionary learning and sparse representation," Multimedia Tools and Applications, vol. 78, no. 24, pp. 34459-34482, 2019.

[23] A. G. Soundari and V. L. Jyothi, "Energy efficient machine learning technique for smart data collection in wireless sensor networks," Circuits, Systems, and Signal Processing, vol. 39, no. 2, pp. 1089-1122, 2020.

[24] M. Rida, A. Makhoul, H. Harb, D. Laiymani, and M. Barhamgi, "EK-means: a new clustering approach for datasets classification in sensor networks," Ad Hoc Networks, vol. 84, pp. 158-169, 2019.

[25] S. Y. Lin, J. B. Li, and C. T. Yu, "Dynamic data driven-based automatic clustering and semantic annotation for internet of things sensor data," Sensors and Materials, vol. 31, no. 6, pp. 1789-1801, 2019.

[26] M. K. Alam, A. A. Aziz, S. A. Latif, and A. Awang, "Data clustering technique for in-network data reduction in wireless sensor network," in 2019 IEEE Student Conference on Research and Development (SCOReD), Bandar Seri Iskandar, Malaysia, October 2019.

[27] D. Ruby and J. Jeyachidra, "Semaphore based data aggregation and similarity findings for underwater wireless sensor networks," International Journal of Grid and High Performance Computing, vol. 11, no. 3, pp. 59-76, 2019.

[28] R. Wan, N. Xiong, Q. Hu, H. Wang, and J. Shang, "Similarityaware data aggregation using fuzzy c-means approach for wireless sensor networks," EURASIP Journal on Wireless Communications and Networking, vol. 2019, no. 1, Article ID 59, 2019.

[29] H. Harb and A. Makhoul, "Energy-efficient scheduling strategies for minimizing big data collection in cluster-based sensor networks," Peer-to-Peer Networking and Applications, vol. 12, no. 3, pp. 620-634, 2019.

[30] R. Nawaz Jadoon, W. Y. Zhou, I. A. Khan, M. A. Khan, and W. Jadoon, "EEHRT: energy efficient technique for handling redundant traffic in zone-based routing for wireless sensor networks," Wireless Communications and Mobile Computing, vol. 2019, Article ID 7502140, 12 pages, 2019.

[31] L. Yang, H. Zhu, H. Wang, K. Kang, and H. Qian, "Data censoring with network lifetime constraint in wireless sensor networks," Digital Signal Processing, vol. 92, pp. 73-81, 2019.

[32] A. Jarwan, A. Sabbah, and M. Ibnkahla, "Data transmission reduction schemes in WSNs for efficient IoT systems," IEEE Journal on Selected Areas in Communications, vol. 37, no. 6, pp. 1307-1324, 2019.

[33] J. Tan, W. Liu, M. Xie et al., "A low redundancy data collection scheme to maximize lifetime using matrix completion technique," EURASIP Journal on Wireless Communications and Networking, vol. 2019, no. 1, Article ID 5, 2019.

[34] K. K. Lim, J. S. Park, and J. G. Shon, "Differential data processing technique to improve the performance of wireless sensor networks," The Journal of Supercomputing, vol. 75, no. 8, pp. 4489-4504, 2019.

[35] S. Madden, "Intel Berkeley Research Lab,” 2004, http://db.csail .mit.edu/labdata/labdata.html. 